\title{
Knockdown of ZFPL1 results in increased autophagy and autophagy-related cell death in NCI-N87 and BGC-823 human gastric carcinoma cell lines
}

\author{
YONG-ZHENG XIE, WAN-LI MA, JI-MING MENG and XUE-QUN REN \\ Department of General Surgery, Huaihe Hospital of Henan University, Kaifeng, Henan 475000, P.R. China
}

Received October 1, 2015; Accepted September 28, 2016

DOI: $10.3892 / \mathrm{mmr} .2017 .6300$

\begin{abstract}
Macroautophagy, which will hereafter be referred to as autophagy, is an evolutionarily conserved process, during which cells recycle and remove damaged organelles and proteins in response to cellular stress. However, the mechanisms underlying the regulation of autophagy remain to be fully elucidated. The present study demonstrated that knockdown of zinc finger protein like 1 (ZFPL1) induces autophagy and increases autophagic cell death in NCI-N87 and BGC-823 human gastric carcinoma cell lines. To examine the role of ZFPL1 in gastric carcinoma cells, ZFPL1 expression was downregulated by lentiviral infection. Zinc finger domain-FLAG was used to compete with ZFPL1 for golgin A2/GM130 binding. Autophagy was analyzed by red fluorescent protein-microtubule-associated protein 1A/1B-light chain 3 (LC3) puncta, LC3I to LC3II conversion, and p62 expression. The results demonstrated that knockdown of ZFPL1 was able to significantly increase cell death rate. However, ZFPL1 knockdown exerted almost no effect on the expression of apoptosis-associated markers, including B cell lymphoma 2 (Bcl-2), Bcl-x, Bcl-2-associated X protein, BH3 interacting domain death agonist, p53, and the classical caspase family members, caspase- 3 , caspase- 8 and caspase- 9 . An endogenous ZFPL1-GM130 association was identified in NCI-N87 cells and BGC-823 cells by co-immunoprecipitation . Furthermore, cell death was restricted following treatment of ZFPL1 knockdown cells with an autophagy inhibitor. Therefore, knockdown of ZFPL1 expression may induce cell death via autophagy, rather than apoptosis. These results suggest that ZFPL1 may serve an important role in regulating autophagy in NCI-N87 and BGC-823 cells.
\end{abstract}

Correspondence to: Dr Xue-Qun Ren, Department of General Surgery, Huaihe Hospital of Henan University, 8 Baobei Road, Kaifeng, Henan 475000, P.R. China

E-mail: xuequnren@163.com

Key words: zinc finger protein like 1, autophagy, cell death, gastric carcinoma cell

\section{Introduction}

Gastric cancer (GC) is the fifth most common cancer worldwide, and is currently the third leading cause of cancer-associated mortality. GC is particularly prevalent in Asia (1). Unfortunately, the majority of patients with GC present with late stage cancer, and therefore require palliative chemotherapy (2). Treatment of GC continues to present a challenge, particularly with regards to the high mortality-to-incidence ratio, despite significant progress being made in early detection and treatment (3). Genetic alterations are thought to be important factors in the vast majority of solid tumors. Recently, gene therapy has been considered an attractive therapeutic option. In addition, autophagy-related genes have attracted attention as novel potential targets in cancer treatment $(4,5)$.

Autophagy is a dynamic process, during which cells recycle and remove damaged organelles and proteins, in order to ensure cell survival in response to cellular stress (6). Furthermore, autophagy can induce cell death under certain conditions. Therefore, the two opposing effects of autophagy serve an important role in cellular differentiation, developmental processes and human disease (7). Several proteins are involved in the detection of autophagic activity, including microtubule-associated protein 1A/1B-light chain 3 (LC3) and the LC3 binding protein, sequestome 1/p62 (8).

The significance of autophagy in cell cycle progression and cell death has previously been reported. A previous study demonstrated that activated autophagy contributes to matrine-induced cell death of the SGC-7901 GC cell line (9). Conversely, autophagy-mediated high mobility group box 1 release promotes GC cell survival via receptor for advanced glycation endproducts activation of extracellular signal-regulated kinases 1/2 (10). However, the function of autophagy in GC remains to be elucidated.

Previous studies indicated the potential roles of zinc finger proteins in gastric cancer progression and the association with autophagy (11). The importance of zinc finger protein like 1 (ZFPL1) in autophagy and human GC resulted in the aim of the present study; to explore the potential antitumor effects of ZFPL1 knockdown, using short hairpin (sh)RNA against ZFPL1 (shZFPL1). The present study explored whether knockdown of ZFPL1 would increase autophagy, inhibit protein glycosylation and promote autophagy-related cell death. In addition, the present study evaluated whether the effects of 
ZFPL1 on autophagy correlated with the interaction between ZFPL1 and GM130.

\section{Materials and methods}

Reagents and antibodies. The MTT Cell Proliferation and Cytotoxicity Assay kit was purchased from Beyotime Institute of Biotechnology (Haimen, China). Immunoblotting was performed using anti-ZFPL1 (cat. no. sc-515393; Santa Cruz Biotechnology, Inc., Dallas, TX, USA), anti- $\beta$-actin (cat. no. sc-47778; Santa Cruz Biotechnology, Inc.), anti-LC3 (cat. no. L8918; Sigma-Aldrich; Merck Millipore, Darmstadt, Germany), anti-p62 (cat. no. N1163; Sigma-Aldrich; Merck Millipore), cleaved caspase antibody sampler kit (cat. no. 2855; Cell Signaling Technology, Inc., Danvers, MA, USA), anti-RL2 (cat.no.SAB1304907;igma-Aldrich;Merck Millipore), anti-p53 (cat. no. ab1431; Abcam, Cambridge, MA, USA), anti-BH3 interacting domain death agonist (Bid; cat. no. ab32060; Abcam), anti-B-cell lymphoma 2 (Bcl-2)-associated X protein (Bax; cat. no. 554104; BD Pharmingen, San Diego, CA, USA), anti-Bcl-x (cat. no. 551269; BD Pharmingen) and anti-Bcl-2 (cat. no. 610539; BD Biosciences, San Jose, CA, USA). Horseradish peroxidase (HRP)-conjugated secondary antibodies (cat. no. 58203) were purchased from Cell Signaling Technology, Inc. Brefeldin A (BFA) and bafilomycin A1 were purchased from Sigma-Aldrich; TRIzol reagent was obtained from Invitrogen (Thermo Fisher Scientific, Inc., Waltham, MA, USA); RevertAid First Strand cDNA Synthesis kit was purchased from Thermo Fisher Scientific, Inc.; and TransStart Green qPCR SuperMix was from Beijing Transgen Biotech Co., Ltd. (Beijing, China). All other reagents were of analytical grade.

Cell culture and lentiviral infection. The NCI-N87 and BGC-823 human GC cell lines were purchased from the American Type Culture Collection (Manassas, VA, USA). The NCI-N87 and BGC-823 cells were cultured in RPMI-1640 medium (Gibco; Thermo Fisher Scientific, Inc.) supplemented with $10 \%$ fetal bovine serum (Gibco; Thermo Fisher Scientific, Inc.), $100 \mu \mathrm{g} / \mathrm{ml}$ streptomycin and $100 \mathrm{U} / \mathrm{ml}$ penicillin at $37^{\circ} \mathrm{C}$ in a humidified atmosphere containing $5 \% \mathrm{CO}_{2}$. Autophagy was induced by culturing cells in medium supplemented with Hanks' balanced salt solution (HBSS; ph 7.2-7.3) or BFA $(1 \mu \mathrm{g} / \mathrm{ml})$ overnight $\left(3 \% \mathrm{CO}_{2}, 37^{\circ} \mathrm{C}\right)$ prior to lentiviral infection. The recombinant plasmids were constructed according to our previous study (12). Transient transfections were performed using FUGENE 6 (Roche Diagnostics, Basel, Switzerland) according to the manufacturer's instructions. The ZFPL1 overexpression was performed using ZFPL1 mimics (cat. no. HMI0070; Sigma-Aldrich; Merck Millipore). RNA interference was carried out using shRNA. A shRNA sequence targeting Lamin A (5'-AAC TGG ACT TCC AGA AGA ACA-3'; Gibco; Thermo Fisher Scientific, Inc.) was used as a negative control. ZFPL1 was targeted with two oligonucleotides (obtained from Gibco; Thermo Fisher Scientific, Inc.): shZFPL1\#1 (5'-CGA CCC GCC TTG TCT GCT A-3') and shZFPL1\#2 (5'-GCT CCA AGA TAG CGA CTA C-3'). All shRNA sequences were cloned into pSUPER.retro.puro vectors (Shanghai Ling Feng Chemical Reagent Co., Ltd., Shanghai, China) for further use. The results presented in the current study were obtained using shZFPL1\#1; shZFPL1\#2 worked similarly. For lentiviral transduction, human embryonic kidney (HEK)293T cells (Shanghai Ling Feng Chemical Reagent Co., Ltd.) were cultured in RPMI-1640 medium (Gibco; Thermo Fisher Scientific, Inc.) supplemented with $10 \%$ fetal bovine serum (Gibco; Thermo Fisher Scientific, Inc.), $100 \mu \mathrm{g} / \mathrm{ml}$ streptomycin and $100 \mathrm{U} / \mathrm{ml}$ penicillin at $37^{\circ} \mathrm{C}$ in a humidified atmosphere containing $5 \% \mathrm{CO}_{2}$. When $75-80 \%$ confluent, the HEK293T cells were transduced (incubated at $3 \% \mathrm{CO}_{2}, 37^{\circ} \mathrm{C}, 25 \mathrm{~min}$ ) with the third-generation packaging plasmids (Cell Signaling Technology, Inc.) pMD2. VSVG, pRSV-REV and pMDLg/pRRE, alongside the transfer constructs. The fresh supernatant was filtered using a $0.45 \mu \mathrm{m}$ filter for further use. The first harvest pool was placed into $50 \mathrm{ml}$ tubes and stored at $4^{\circ} \mathrm{C}$ and then centrifuged for $10 \mathrm{~min}$ at $1,153 \times \mathrm{g}$ to produce a pellet consisting of cells and debris. The cell-free supernatant was subjected to ultra-centrifugation for $120 \mathrm{~min}\left(16^{\circ} \mathrm{C}\right)$ at $49,460 \times \mathrm{g}$, after which the supernatant was discarded and the pellet was resuspended in HBS. The vector stock solutions were pooled to produce a homogenous vector stock solution and stored at $-80^{\circ} \mathrm{C}$. Freezing and thawing of the lentiviral stock were avoided as much as possible. Infected NCI-N87 and BGC-823 cells were rinsed with PBS, and were allowed to recover for $12 \mathrm{~h}$ prior to further experimentation.

Cell growth inhibition assay. Cell growth inhibition was detected using the MTT Cell Proliferation and Cytotoxicity Assay kit (Siemens AG, Munich, Germany). The present study consisted of the following four groups: Control shRNA group, shZFPL1 group, control shRNA + bafilomycin A1 group and shZFPL1 + bafilomycin A1 group. Bafilomycin A1 group cells were incubated with $3 \mathrm{mg} / \mathrm{ml}$ bafilomycin A1 at room temperature for $3 \mathrm{~h}$. Briefly, cells in the logarithmic growth phase were collected and reseeded into 96-well plates at 10,000 cells/well. Following a $24 \mathrm{~h}$ incubation, the medium was gently removed from all wells and fresh medium containing $10 \mu \mathrm{l}$ MTT $(5 \mathrm{mg} / \mathrm{ml})$ was added to each well. Following a further $4 \mathrm{~h}$ incubation at $37^{\circ} \mathrm{C}, 150 \mu \mathrm{l}$ dimethyl sulfoxide (Sigma-Aldrich; Merck Millipore) was added, and the plates were slowly agitated for $10 \mathrm{~min}$ at room temperature. Subsequently, Sorensen's glycine buffer ( $0.1 \mathrm{M}$ glycine, $0.1 \mathrm{M} \mathrm{NaCl} ; \mathrm{pH} 10.5$ ) was added, and the absorbance of each well was measured at $490 \mathrm{~nm}$ in $10 \mathrm{~min}$. The cell growth inhibition rate was calculated as follows: $(1$ - experimental group absorbance/control group absorbance) x100. A graph was plotted using GraphPad Prism 5 software (GraphPad Software, Inc., La Jolla, CA, USA). All experiments were carried out in triplicate.

Western blotting. The cells were harvested in PBS, and were homogenized in lysis buffer [ $25 \mathrm{mM}$ Tris- $\mathrm{HCl}$ (pH 7.4), $5 \mathrm{mM}$ $\mathrm{MgCl}_{2}, 200 \mathrm{mM} \mathrm{NaCl}, 1 \mathrm{mM}$ EDTA, $2 \mathrm{mM} \mathrm{Na}_{3} \mathrm{VO}_{4}, 0.1 \mathrm{mM}$ EGTA, $1 \%$ NP-40, $0.1 \%$ SDS, $10 \%$ glycerol, 1 mM phenylmethylsulfonyl fluoride (cat. no. 8553; Cell Signaling Technology, Inc.) and Protease Inhibitor Cocktail (cat. no. 5871; Cell Signaling Technology, Inc.)] on ice for $30 \mathrm{~min}$. Lysates were then centrifuged at $28,000 \times g$ for $30 \mathrm{~min}$ at $4^{\circ} \mathrm{C}$. The supernatants were carefully transferred into fresh Eppendor ${ }^{\circledR}$ tubes and the protein concentration was detected using the Bradford method. Subsequently, the protein samples were 
denatured at $100^{\circ} \mathrm{C}$ for $10 \mathrm{~min}$ and were cooled on ice. Protein samples $(35 \mu \mathrm{g})$ were separated by $10-15 \%$ SDS-PAGE $(150 \mathrm{~V}$, $70 \mathrm{~min}$ ) and were transferred to a polyvinylidene difluoride membrane (90 V, 60 min). After blocking with 5\% nonfat milk in TBS- $0.1 \%$ Tween 20 for $1 \mathrm{~h}$ at room temperature, the PVDF membranes were blotted with primary antibodies (1:500 dilution) overnight at $4^{\circ} \mathrm{C}$. Subsequently, the membranes were incubated with HRP-conjugated secondary antibodies $(1: 1,000$ dilution) at room temperature for $1 \mathrm{~h}$. After washing, the band intensity was analyzed using HYBOND-ECL (Sangon Biotech Co., Ltd., Shanghai, China) and the LAS-3000 luminescent image analyzer system (Fujifilm, Tokyo, Japan).

Co-immunoprecipitation. Co-immunoprecipitation experiments were performed as previously described (12).

Reverse transcription-quantitative polymerase chain reaction (RT-qPCR). Total RNA was extracted from the cells using TRIzol ${ }^{\circledR}$ reagent, according to the manufacture's protocol. RNA $(4 \mu \mathrm{g})$ then underwent RT-qPCR. The reaction mixture contained $4 \mu 1$ RNA, $4 \mu 15^{*}$ PrimeScript Buffer, $1 \mu 1$ PrimerScript RT Enzyme Mix I (Applied Biosystems; Thermo Fisher Scientific, Inc.), $1 \mu \mathrm{l}$ oligo dT primer and $1 \mu \mathrm{l}$ random hexamers in a total of $20 \mu \mathrm{l}$. The primers used were as follow: GAPDH, forward 5'-AAG CTC ATT TCC TGG TAT GAC AAC G-3', reverse 5'-TCT TCC TCT TGT GCT CTT GCT GG-3', which served as an internal reference gene (126 bp); and ZFPL1, forward 5'-GCC GAT CAG TAA ACA CAG A-3' and reverse 5'-ACT GGA CGA TGC ACT TG-3' (149 bp). The amplification conditions were as follows: Pre-denaturation at $95^{\circ} \mathrm{C}$ for $5 \mathrm{~min}$, followed by 40 cycles of denaturation at $95^{\circ} \mathrm{C}$ for $30 \mathrm{sec}$, annealing at $58^{\circ} \mathrm{C}$ for $30 \mathrm{sec}$ and extension at $72^{\circ} \mathrm{C}$ for $30 \mathrm{sec}$, and a final extension at $72^{\circ} \mathrm{C}$ for $5 \mathrm{~min}$. Changes in ZFPL1 expression levels were calculated using the $2^{-\Delta \Delta \mathrm{Cq}}$ method (13). Each sample was tested in triplicate.

Microscopy. Cells stably expressing red fluorescent protein (RFP)-LC3 plasmid (Addgene, Inc., Cambridge, MA, USA) were cultured in a $25-\mathrm{cm}^{2}$ culture bottle. A fluorescence microscope (Nikon Corporation, Tokyo, Japan) was used to observe cells expressing RFP. Images were obtained using a charge-coupled device camera (Coolsnap; Nikon Corporation) with MetaMorph 7.8 software (Molecular Devices, LLC, Sunnyvale, CA, USA). At least 200 cells from 10 different fields were counted for statistical analysis.

Statistical analysis. All statistical analyses were conducted using GraphPad Prism 5.0 software. Data were analyzed using one-way analysis of variance and least significant difference test. $\mathrm{P}<0.05$ was considered to indicate a statistically significant difference.

\section{Results}

ZFPL1 expression is decreased and cell death is increased in autophagy-activated cells. It is well known that BFA and nutrient deprivation induce autophagy in several cell lines. In the present study, ZFPL1 expression was decreased in NCI-N87 cells following treatment with HBSS or BFA $(1 \mu \mathrm{g} / \mathrm{ml})$; similar results were observed in another human
GC cell line, BGC-823 (Fig. 1A-C). In addition, the cell death rate was markedly increased in HBSS- or BFA-treated cells compared with in the control group (Fig. 1D).

Knockdown of ZFPL1 induces autophagy in NCI-N87 and BGC-823 cells. To determine the effects of ZFPL1 on autophagy regulation, a ZFPL1 knockdown system was generated using lentiviral infection. To investigate the silencing efficiency of two shZFPL1 shRNA molecules, western blotting was performed in NCI-N87 and BGC-823 cells. The results demonstrated that shZFPL1 significantly decreased ZFPL1 protein levels compared with the control group, as detected by western blotting and densitometric analysis (Fig. 2A and B). The marked decrease in ZFPL1 mRNA expression was further confirmed by qPCR (Fig. 2C). It has previously been reported that ZFPL1 directly interacts with the cis-Golgi matrix protein golgin A2/GM130 (14), which is a key factor in stacking of Golgi cisternae and Golgi structural maintenance (15). Previous studies have indicated that Golgi and autophagy-related membrane trafficking are functionally interdependent (16). In addition, autophagosome formation has been detected in GM130-downregulated cells (17). To determine whether ZFPL1 was associated with autophagy, LC3-I to LC3-II conversion and p62 degradation were detected by western blotting and densitometric analysis in ZFPL1 knockdown cells. LC3-I and LC3-II are cellular forms of the LC3 protein; LC3-I is the cytoplasmic form, whereas LC3-II is the autophagosome membrane-bound form $(18,19)$. Therefore, an increase in the ratio of LC3-II to LC3-I protein is correlated with the extent of autophagosome formation. p62, which is a ubiquitin-binding scaffold protein, is associated with autophagy by directly binding to the autophagic effector proteins gamma-aminobutyric acid receptor-associated protein and LC3 for degradation (20). Therefore, total cellular levels of p62 reflect autophagic activity. The present study demonstrated that knockdown of ZFPL1 significantly increased the ratio of LC3-II to LC3-I and p62 degradation, as compared with the shRNA control (Fig. 2D and E). In the present study, RFP-LC3 was examined in shZFPL1 NCI-N87 and BGC-823 cells, and autophagy induction was evaluated using confocal microscopy. As shown in Fig. 2F, shZFPL1 cells exhibited an increase in LC3 puncta compared with the control cells. These results clearly indicate that downregulation of ZFPL1 induces autophagy in NCI-N87 and BGC-823 cells.

Effects of ZFPL1 knockdown on autophagy and glycosylation in NCI-N87 and BGC-823 cells. The cis-Golgi matrix protein GM130, which directly interacts with ZFPL1, serves an essential role in protein glycosylation, and downregulation of GM130 decreases the level of RL2 (17). To determine the effects of ZFPL1 knockdown on protein glycosylation, the expression levels of RL2 in ZFPL1 knockdown cells were detected by western blotting. The expression of RL2 was markedly decreased in a time-dependent manner, whereas the ratio of LC3II to LC3I was increased in stably transfected cells treated with BFA (Fig. 3A and B). To further confirm the function of ZFPL1 on protein glycosylation regulation, a ZFPL1 overexpression assay was conducted using a lentiviral system. Notably, the expression levels of RL2 and the ratio of LC3II to LC3I were reversed in cells overexpressing ZFPL1 compared 

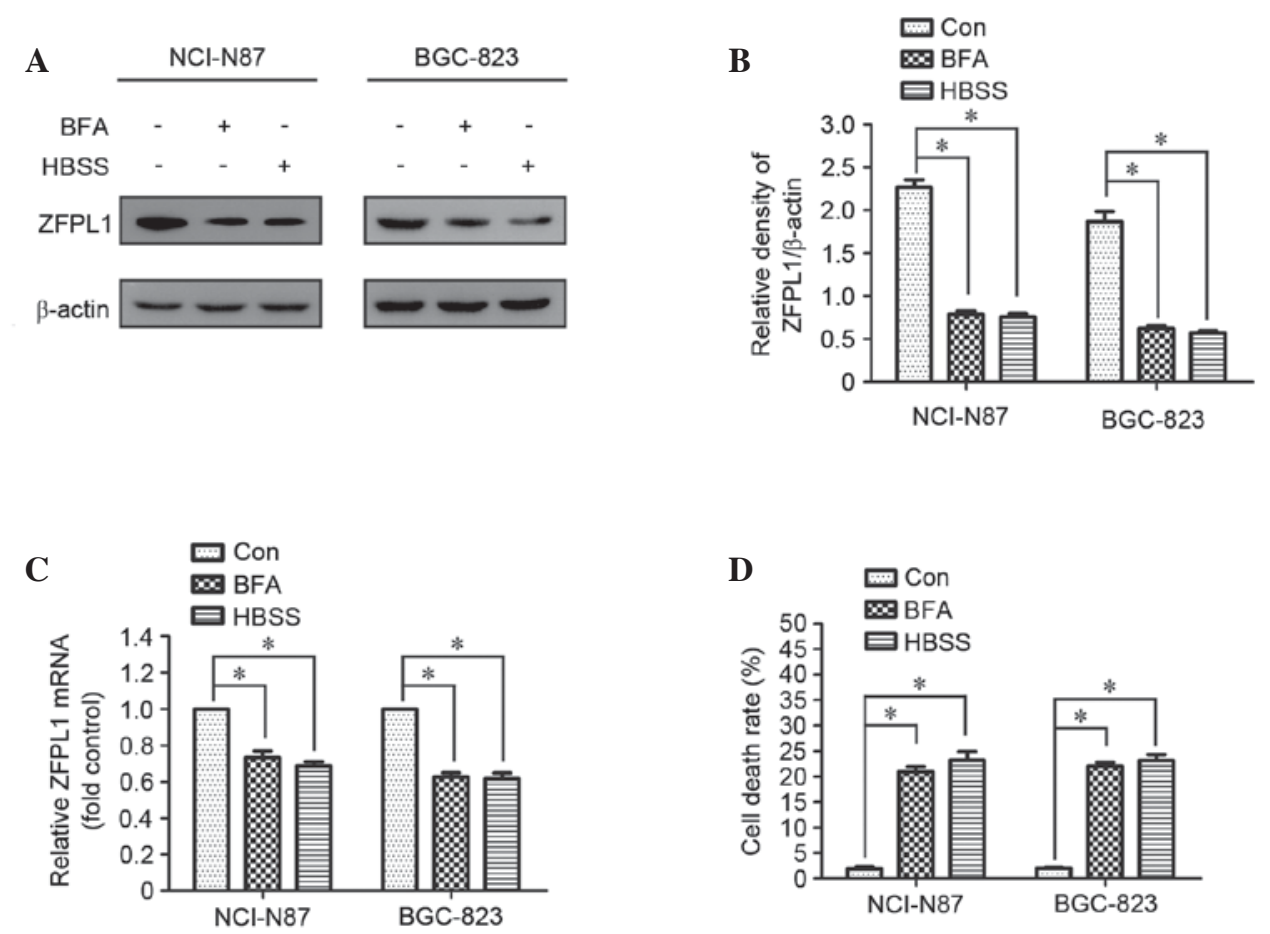

Figure 1. ZFPL1 expression was decreased and cell death was increased in autophagy-activated cells. NCI-N87 and BGC-823 cells were treated with BFA $(1 \mu \mathrm{g} / \mathrm{ml})$ or HBSS for $2 \mathrm{~h}$. (A) Total cell lysates were analyzed using anti-ZFPL1 antibody to detect ZFPL1 expression. $\beta$-actin was used as a loading control. (B) ZFPL1 protein levels shown in (A) were measured by densitometric analysis of protein bands and were normalized to $\beta$-actin (n=3). (C) Reverse transcription-quantitative polymerase chain reaction analysis of relative ZFPL1 mRNA expression. The mRNA expression levels were normalized to GAPDH and presented as a fold change compared with the control cells $(n=3)$. (D) Treatment with HBSS or BFA induced cell death. Cell death rate was detected using the MTT assay, $(\mathrm{n}=3)$. Data are presented as the mean \pm standard error of the mean of three independent replicates. ${ }^{*} \mathrm{P}<0.05$. ZFPL1, zinc finger protein like 1; Con, control; BFA, brefeldin A; HBSS, Hanks' balanced salt solution.

with ZFPL1 knockdown cells (Fig. 3C and D). These results suggest that knockdown of ZFPL1 may induce autophagy and reduce the protein glycosylation process.

ZFPL1 knockdown induces autophagy-associated cell death, rather than apoptosis, in NCI-N87 and BGC-823 cells. Autophagy is an important mechanism of cell survival in response to stress conditions, including nutrient deprivation, reactive oxygen species and ursulic acid; however, autophagic dysfunction also leads to cell death, which is known as autophagic cell death or type II programmed cell death $(21,22)$. The present study demonstrated that cell death was significantly increased in ZFPL1 knockdown NCI-N87 and BGC-823 cells. However, in ZFPL1 knockdown cells treated with bafilomycin $\mathrm{A} 1(10 \mu \mathrm{M})$, cell death was markedly reduced compared with in the untreated cells (Fig. 4A). Immunoblotting analysis indicated that transfection with shZFPL1 or control shRNA did not affect the expression of apoptosis-associated proteins, including Bcl-2, Bcl-x, Bax, Bid, p53, and the classical caspase family members, caspase-3, caspase- 8 and caspase-9 (Fig. 4B and C). Conversely, the induction of autophagy by ZFPL1 knockdown was confirmed using the autophagy inhibitor bafilomycin A1, which blocks the fusion process of autophagosomes and mature lysosomes (23). Western blotting indicated that the expression levels of LC3II, the autophagy biomarker, increased following knockdown of ZFPL1 (Fig. 2D and E). As shown in Fig. 2E, the ratio of LC3II to LC3I was increased following knockdown of ZFPL1 compared with the control. The autophagy inhibitor bafilomycin A1 was used to determine the effects of ZFPL1 knockdown on autophagy. Cells stably transfected with shZFPL1 and treated with bafilomycin A1 $(10 \mu \mathrm{M})$ exhibited no difference in LC3 expression compared with the control cells (Fig. 4D and E). These results suggest that ZFPL1 knockdown may induce autophagy-related cell death in NCI-N87 and BGC-823 cells.

Effects of ZFPL1 on autophagy are associated with the interaction between ZFPL1 and GM130. To determine whether the interaction between ZFPL1 and GM130 affects autophagy, a co-immunoprecipitation assay was conducted. An endogenous ZFPL1-GM130 association was identified in NCI-N87 cells (Fig. 5A); similar results were observed in BGC-823 cells (data not shown). Chiu et al (14) revealed that the first zinc finger domain (ZFD; 1-43 amino acid) of ZFPL1 contained the GM130-binding site. Therefore, the present study overexpressed ZFD-FLAG to further analyze the effects of ZFPL1-GM130 interaction on autophagy. As shown in Fig. 5B and C, ZFD-FLAG competed with ZFPL1 for GM130 binding; however, no effect on ZFPL1 expression was observed in the process. The results of the present study indicated that ZFPL1 knockdown induced autophagy in NCI-N87 and BGC-823 cells. Therefore, knockdown of ZFPL1 should result in decreased endogenous ZFPL1-GM130 association. In addition, autophagy was induced after using competitive binding reactions with ZFPL1 and ZFD-Flag (Fig. 5C and D). These results suggest that ZFPL1 regulates autophagy via a GM130-interacting mechanism. 
A

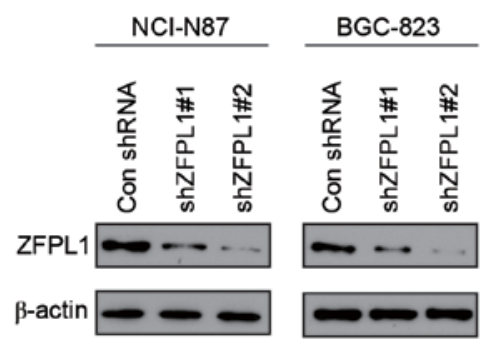

\section{C $\quad$ Con shRNA $\mathbb{~ s h Z F P L 1 \# 1 ~}$ 口 shZFPL1\#2}

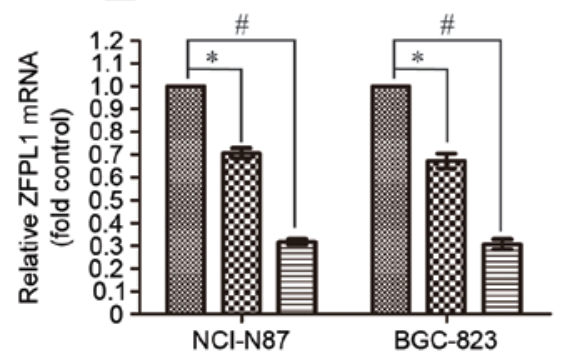

$\mathbf{E}$

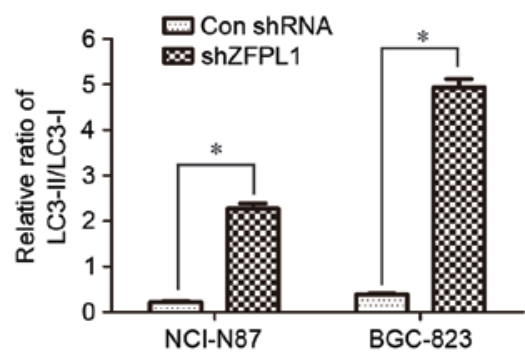

G

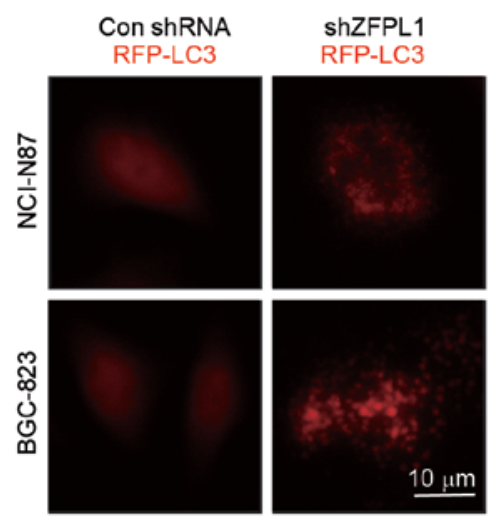

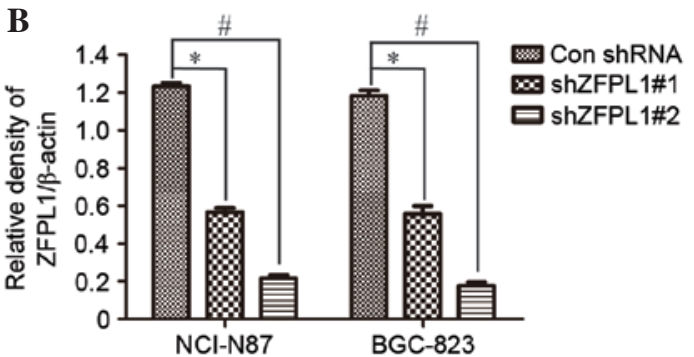

D

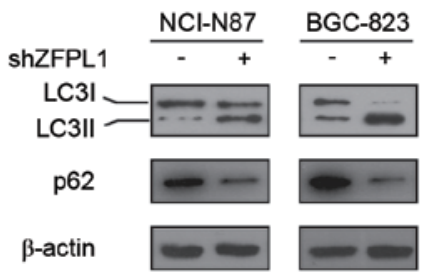

$\mathbf{F}$

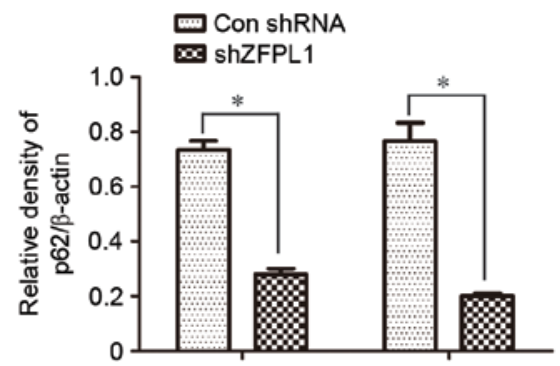

H

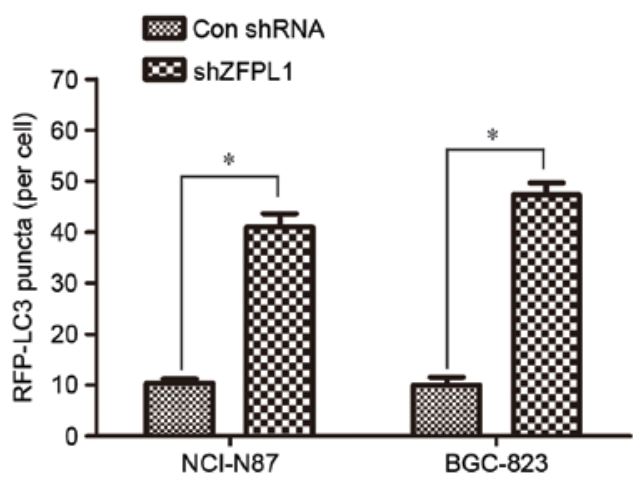

Figure 2. Knockdown of ZFPL1 induces autophagy in NCI-N87 and BGC-823 cells. (A) ZFPL1 knockdown efficiency of shRNA\#1 and 2, as well as control shRNA against lamin A, as determined by western blotting. (B) Densitometric analysis normalized to $\beta$-actin. (C) Quantitative polymerase chain reaction analysis normalized to GAPDH demonstrated that RNA interference decreased ZFPL1 mRNA levels. (D) LC3, p62 and $\beta$-actin were analyzed by western blotting. (E) LC3II/LC3I and (F) p62/ß-actin levels were measured using densitometric analysis. (G) Representative fluorescence microscope images of NCI-N87 and BGC-823 cells stably expressing RFP-LC3. Scale bar: $10 \mu \mathrm{m}$. (H) Average number of RFP-LC3 puncta per cell was counted in $>5$ fields with $\geq 100$ cells per group. All quantitative data are presented as the mean \pm standard error of the mean of $\geq 3$ independent experiments. "P $<0.05$, and "P<0.01. ZFPL1, zinc finger protein like 1; shRNA, short hairpin RNA; LC3, microtubule-associated protein 1A/1B-light chain 3; RFP, red fluorescent protein; Con, control.

\section{Discussion}

GC is the fifth most common cancer globally, and is the third leading cause of cancer-associated mortality; $>723,000$ individuals succumb to GC annually, despite significant progress in early detection and improvements to systemic cytotoxic chemotherapy regimens (1). The high mortality rate associated with GC is predominantly due to its silent nature, advanced stage, and underlying biological and genetic heterogeneity. A more detailed molecular understanding of the GC pathogenesis is required to improve patient outcomes. Recent research regarding biochemical pathways has guided researchers 
A

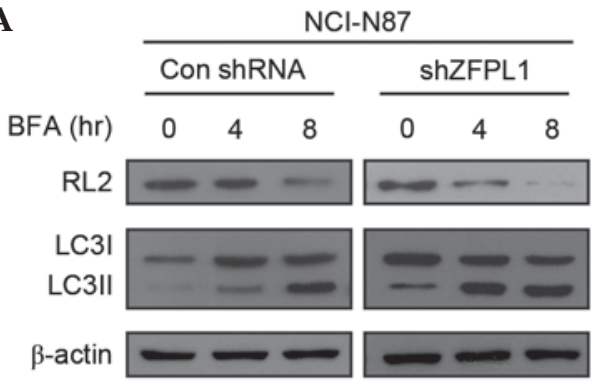

B

NCI-N87

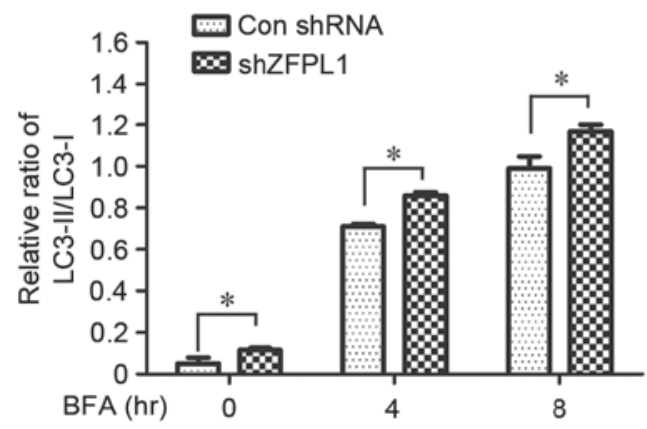

C

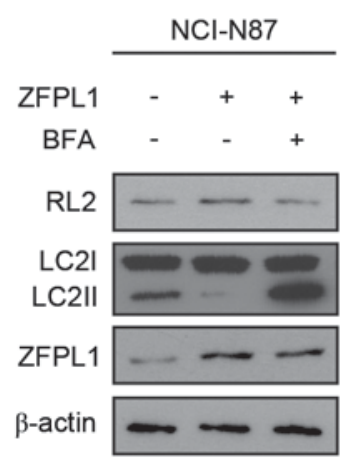

BGC-823

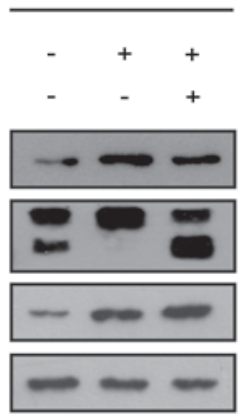

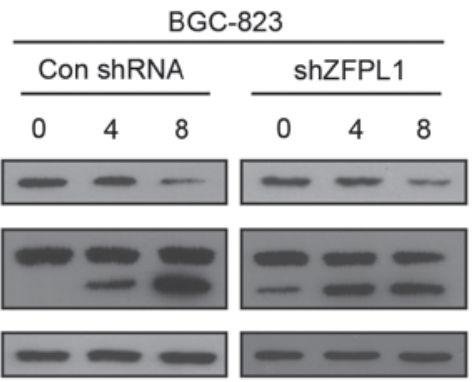

BGC-823

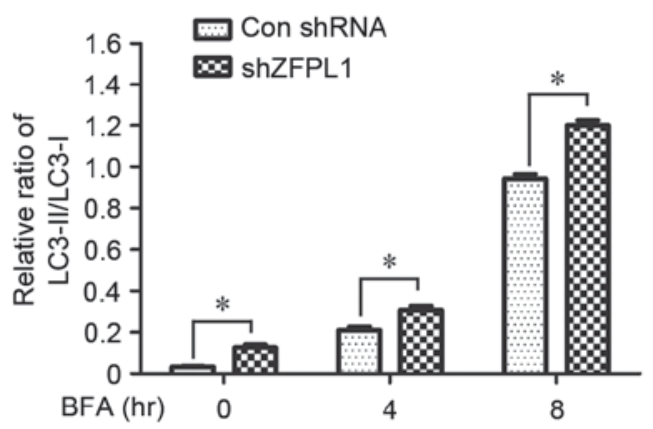

D

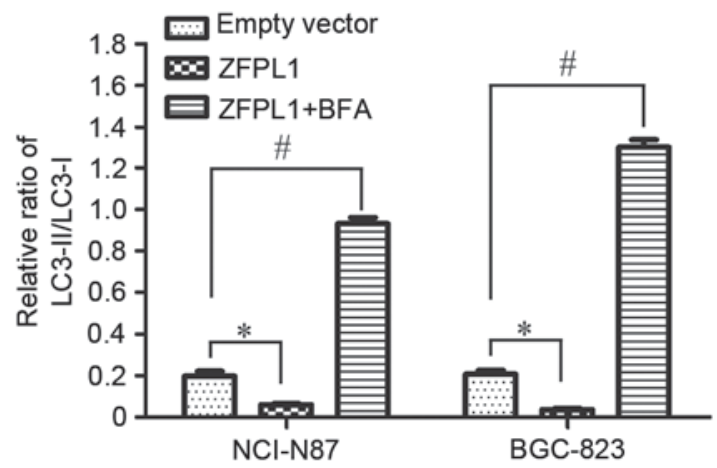

Figure 3. Downregulation of ZFPL1 attenuates protein glycosylation and induces autophagy in NCI-N87 and BGC-823 cells. (A) Western blotting of RL2 and LC3. Cells stably transfected with ZFPL1 shRNA were treated with BFA $(1 \mu \mathrm{g} / \mathrm{ml})$ for 4 or $8 \mathrm{~h}$, whole cell lysates were harvested for western blotting. (B) LC3II/LC3I levels shown in (A) were measured using densitometric analysis. (C) Cells were transfected with ZFPL1 expression or control vector lentiviruses, and stably overexpressed cells were treated with BFA $(1 \mu \mathrm{g} / \mathrm{ml})$ for $8 \mathrm{~h}$. Western blot analysis was used to detect protein expression levels of RL2, ZFPL1 and LC3. (D) LC3II/LC3I levels shown in (C) were measured using densitometric analysis. Data are presented as the mean \pm standard error of the mean of three independent experiments. " $\mathrm{P}<0.05,{ }^{\text {}} \mathrm{P}<0.01$. ZFPL1, zinc finger protein like 1 ; shRNA, short hairpin RNA; LC3, microtubule-associated protein $1 \mathrm{~A} / 1 \mathrm{~B}-$-light chain 3 ; BFA, brefeldin A; Con, control.

to identify potential targets. In cancer therapy, tumor cell death is a vital event in the clearance of cancer cells, and anticancer therapies often involve caspase-dependent apoptosis (24-26). However, it has been indicated that cell death could be categorized to a few types, including 'accidental cell death' (ACD) and 'regulated cell death' (RCD), obviously caspase-dependent apoptosis is one subtype of RCD. In the context of antitumor therapy, autophagic pathways are being considered as potential targets for therapeutic intervention $(27,28)$. Three types of autophagy have been discussed in the field of antitumor therapy, based on their effects on cells: Cytoprotective, nonprotective and cytotoxic. The three forms can not be clearly distinguished via molecular or biochemical characteristics (29). Therefore, autophagy regulation may provide antitumor therapeutic opportunities.

The present study aimed to explore potential anticancer effects by silencing the expression of ZFPL1, and uncovered the mechanisms underlying the effects of ZFPL1 on autophagy regulation. The results demonstrated that ZFPL1 was downregulated when autophagy was activated by HBSS or BFA in NCI-N87 and BGC-823 human gastric cancer cell lines (Fig. 1A). Previous studies regarding autophagy have indicated that functional autophagy serves a central role in cellular recycling, homeostasis maintenance and stress response (30). However, excessive autophagy disturbs cellular energy supply and negatively affects cell survival; 

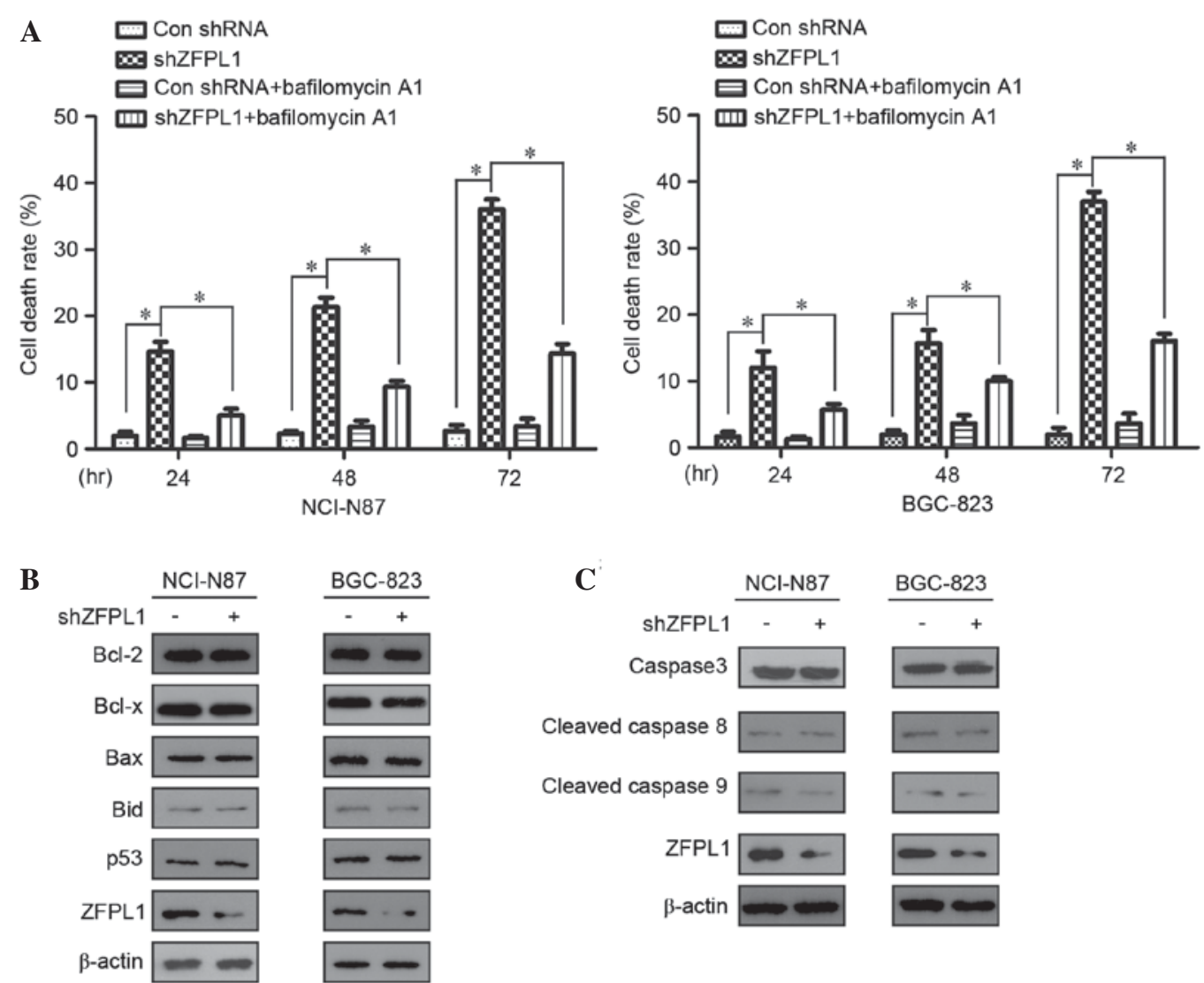

$\mathbf{D}$
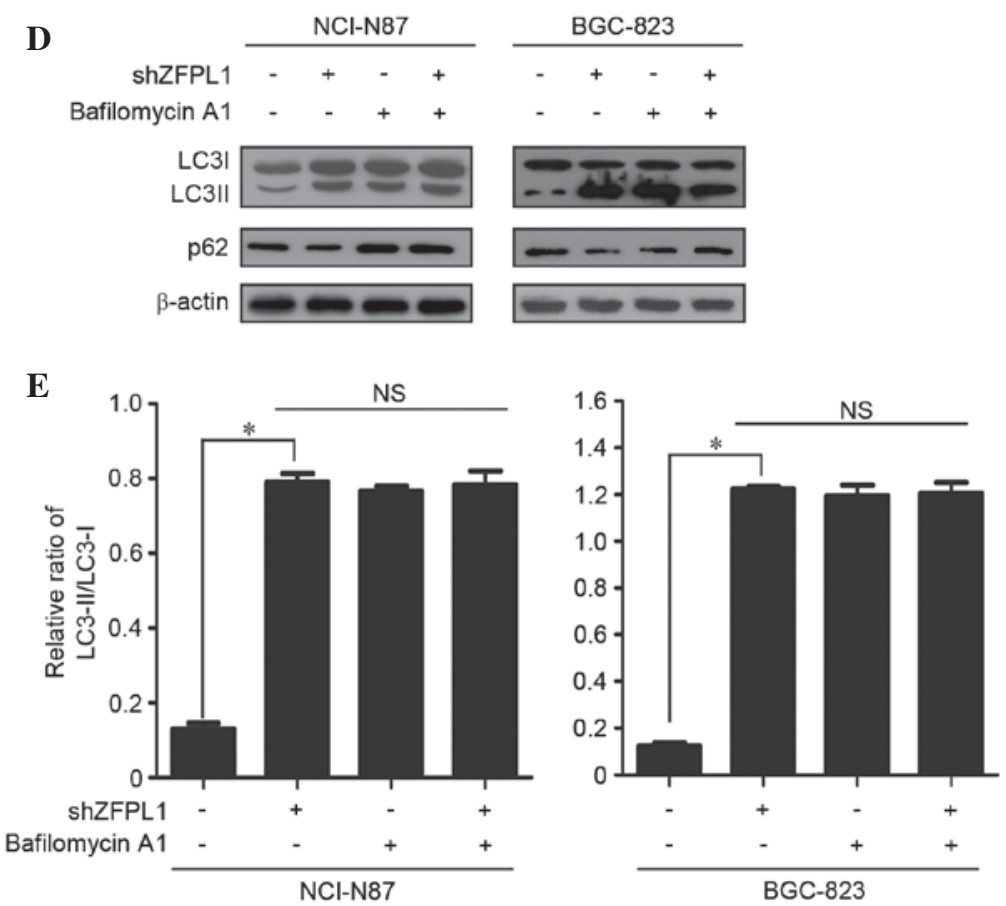

Figure 4. Knockdown of ZFPL1 induces autophagy-related cell death, rather than apoptosis, in NCI-N87 and BGC-823 cells. (A) Cell death rate was measured using the MTT assay $(\mathrm{n}=3)$. Knockdown and control cells were treated with bafilomycin A1 (10 $\mu \mathrm{M})$ for 24, 48 or $72 \mathrm{~h}$. (B and C) Cell lysates were collected post-transfection with shZFPL1 or control shRNA for $48 \mathrm{~h}$, and protein expression was determined using western blotting, with $\beta$-actin as an internal control. (D and E) Knockdown and control cells were treated with bafilomycin A1 $(10 \mu \mathrm{M})$ for $48 \mathrm{~h}$. Western blotting was used to detect LC3 and p62 expression. Data are presented as the mean \pm standard error of the mean of three independent experiments. "P<0.05. NS, no significance; ZFPL1, zinc finger protein like 1; shRNA, short hairpin RNA; Bcl-2, B-cell lymphoma 2; Bax, Bcl-2-associated X protein; Bid, BH3 interacting domain death agonist; LC3, microtubule-associated protein 1A/1B-light chain 3; Con, control.

therefore, autophagic cell death is considered hyperstimulated self-eating $(29,31,32)$. The present study also demonstrated that increased cell death was induced by HBSS or BFA (Fig. 1D). Therefore, it may be hypothesized that cell death is 
A

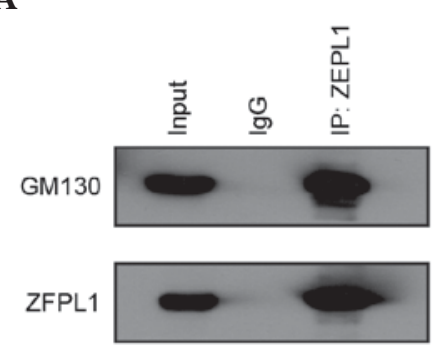

B

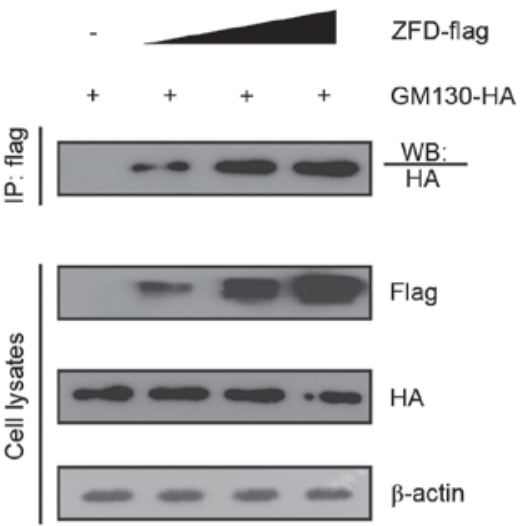

C

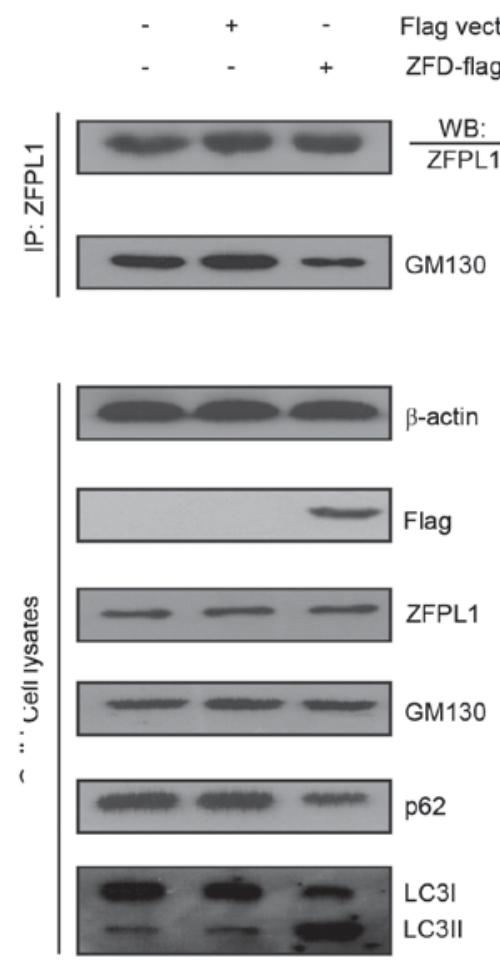

D

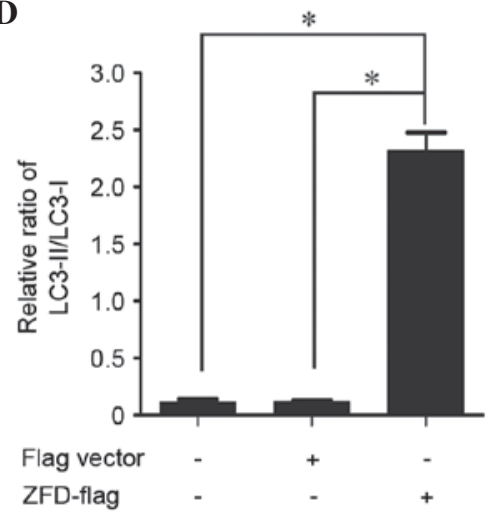

Figure 5. Effects of ZFPL1 on autophagy are associated with the interaction between ZFPL1 and GM130. (A) Endogenous ZFPL1 coimmunoprecipitates with GM130 in NCI-N87 cells. (B) ZFD-FLAG coimmunoprecipitates with HA-GM130. Human embryonic kidney $293 \mathrm{~T}$ cells were grown to 80\% confluence, and were then transiently co-transfected with pCMV HA-GM130 plasmid (6 $\mu \mathrm{g})$ and pcDNA3 ZFD-FLAG plasmid (0, 2, 4 and $6 \mu \mathrm{g})$. (C) ZFD-FLAG competed with ZFPL1 for GM130 binding in NCI-N87 cells. NCI-N87 cells stably expressed FLAG vector or ZFD-FLAG; two thirds of the cell lysate was used for coimmunoprecipitation experiments, and the remaining third was used to assess LC3 and p62 expression. (D) LC3II/LC3I levels were measured using densitometric analysis. Data are presented as the mean \pm standard error of the mean of three independent experiments. ${ }^{*} \mathrm{P}<0.05$. ZFPL1, zinc finger protein like 1 ; shRNA, short hairpin RNA;LC3, microtubule-associated protein 1A/1B-light chain 3; IP, immunoprecipitation; HA, hemagglutinin; IgG, immunoglobulin G; Con, control.

regulated by ZFPL1. This hypothesis is supported by a series of experiments.

The results of the present study demonstrated that ZFPL1 knockdown induces autophagy (Fig. 2D and G), and basic autophagy is mitigated by overexpression of ZFPL1. It has previously been reported that ZFPL1 has the properties of a Golgi matrix protein, and directly interacts with GM130 (14). It is also well known that downregulation of GM130 results in impaired protein glycosylation and active autophagy (17). The majority of mammalian proteins synthesized in the endoplasmic reticulum undergo glycosylation. Glycosylation acts as a modulator of GC cellular behavior, including cell differentiation, cell-cell and cell-matrix interactions, cell-pathogen interactions, invasion, and metastasis (33).
It has previously been observed that glycosylation modifications may be good targets for cancer therapy, such as synthesis of the $\beta 1,6 \mathrm{GlcNAc}$ branched N-glycans, and these modifications appear to be an attractive tool for future applications $(34,35)$. The present study demonstrated that ZFPL1 knockdown was associated with impaired protein glycosylation (Fig. 3A), and overexpression of ZFPL1 could rescue glycosylation (Fig. 3C). Therefore, as a mediator of glycosylation and autophagy, downregulation of ZFPL1 in cancer cells may impair cell survival.

The present study also demonstrated that cell death rate was increased following ZFPL1 knockdown, and treatment with the autophagy inhibitor bafilomycin A1 was able to reduce cell death rate (Fig. 4A). Therefore, it may be hypothesized 
that ZFPL1 knockdown-induced cell death is dependent on autophagy. A series of apoptotic markers were detected using western blotting, and the expression levels of hardly any of these markers were increased (Fig. 4B and C). Autophagy induced by ZFPL1 knockdown was markedly suppressed using bafilomycin A1 (Fig. 4D). These results indicated that ZFPL1 may have an important role in autophagy-related cell death. A previous study observed that GM130 directly binds ZFPL1 (14), and loss of GM130 induces autophagy (17). Therefore, the interaction between ZFPL1 and GM130 may be important in autophagy regulation.

A co-immunoprecipitation analysis was conducted in NCI-N87 cells, and the interaction between ZFPL1 and GM130 was detected, in accordance with previous studies (Fig. 5A) (36). There are two ZFDs at the N-terminus (37), and the first ZFD (1-43 amino acid) has been verified as the GM130-binding region (14). In the present study, ZFD-FLAG competed with ZFPL1 for GM130 binding; however, wild type ZFPL1 hardly inhibits the binding (Fig. 5B and C). Notably, autophagy was activated after ZFPL1 was replaced for GM130 binding by overexpressed ZFD-FLAG. These data suggest that it is possible to induce autophagy-related cell death using drugs that interfere with the association between ZFPL1 and GM130.

In conclusion, the present study demonstrated that ZFPL1 serves as a regulator of autophagy-related cell death, which may explain how ZFPL1 in human GC cells underlies the onset of autophagy. Due to the relevant role of ZFPL1, the reducing the function of ZFPL1 may provide a potential intervention strategy for the treatment of GC.

\section{Acknowledgements}

The present study was supported by a grant from the Science and Technology Develop Project in Kaifeng (project number: 1403005).

\section{References}

1. Tan P and Yeoh KG: Genetics and molecular pathogenesis of gastric adenocarcinoma. Gastroenterology 149: 1153-1162.e3, 2015 .

2. Wesolowski R, Lee C and Kim R: Is there a role for second-line chemotherapy in advanced gastric cancer? Lancet Oncol 10: 903-912, 2009.

3. Takahashi T, Saikawa Y and Kitagawa Y: Gastric cancer: Current status of diagnosis and treatment. Cancers 5: 48-63, 2013.

4. Bhutia SK, Das SK, Azab B, Dash R, Su ZZ, Lee SG, Dent P, Curiel DT, Sarkar D and Fisher PB: Autophagy switches to apoptosis in prostate cancer cells infected with melanoma differentiation associated gene-7/interleukin-24 (mda-7/IL-24). Autophagy 7: 1076-1077, 2011.

5. Janku F, McConkey DJ, Hong DS and Kurzrock R: Autophagy as a target for anticancer therapy. Nat Rev Clin Oncol 8: 528-539, 2011.

6. Klionsky DJ and Emr SD: Autophagy as a regulated pathway of cellular degradation. Science 290: 1717-1721, 2000.

7. Levine B and Kroemer G: Autophagy in the pathogenesis of disease. Cell 132: 27-42, 2008.

8. Klionsky DJ, Codogno P, Cuervo AM, Deretic V, Elazar Z, Fueyo-Margareto J, Gewirtz DA, Kroemer G, Levine B, Mizushima N, et al: A comprehensive glossary of autophagy-related molecules and processes. Autophagy 6 : 438-448, 2010.

9. Zhang J, Li Y, Chen X, Liu T, Chen Y, He W, Zhang Q and Liu S: Autophagy is involved in anticancer effects of matrine on SGC-7901 human gastric cancer cells. Oncol Rep 26: 115-124, 2011.
10. Zhang QY, Wu LQ, Zhang $\mathrm{T}$, Han $\mathrm{YF}$ and Lin $\mathrm{X}$ : Autophagy-mediated HMGB1 release promotes gastric cancer cell survival via RAGE activation of extracellular signal-regulated kinases 1/2. Oncol Rep 33: 1630-1638, 2015.

11. Thedieck K, Holzwarth B, Prentzell MT, Boehlke C, Kläsener K, Ruf S, Sonntag AG, Maerz L, Grellscheid SN, Kremmer E, et al: Inhibition of mTORC1 by astrin and stress granules prevents apoptosis in cancer cells. Cell 154: 859-874, 2013.

12. Sun Y, Liu JH, Jin L, Sui YX, Han LL and Huang Y: Effect of autophagy-related beclin1 on sensitivity of cisplatin-resistant ovarian cancer cells to chemotherapeutic agents. Asian Pac J Cancer Prev 16: 2785-2791, 2015.

13. Livak KJ and Schmittgen TD: Analysis of relative gene expression data using real-time quantitative PCR and the 2(-Delta Delta C(T)) Method. Methods 25: 402-408, 2001.

14. Chiu CF, Ghanekar Y, Frost L, Diao A, Morrison D, McKenzie E and Lowe M: ZFPL1, a novel ring finger protein required for cis-Golgi integrity and efficient ER-to-Golgi transport. EMBO J 27: 934-947, 2008.

15. Nakamura N, Rabouille C, Watson R, Nilsson T, Hui N, Slusarewicz P, Kreis TE and Warren G: Characterization of a cis-Golgi matrix protein, GM130. J Cell Biol 131: 1715-1726, 1995.

16. Keith SA, Maddux SK, Zhong Y, Chinchankar MN, Ferguson AA, Ghazi A and Fisher AL: Graded proteasome dysfunction in Caenorhabditis elegans activates an adaptive response involving the conserved SKN-1 and ELT-2 transcription factors and the autophagy-lysosome pathway. PLoS Genet 12: e1005823, 2016.

17. Chang SH, Hong SH, Jiang HL, Minai-Tehrani A, Yu KN, Lee JH, Kim JE, Shin JY, Kang B, Park S, et al: GOLGA2/GM130, cis-Golgi matrix protein, is a novel target of anticancer gene therapy. Mol Ther 20: 2052-2063, 2012.

18. Klionsky DJ, Abdalla FC, Abeliovich H, Abraham RT, Acevedo-Arozena A, Adeli K, Agholme L, Agnello M, Agostinis P, Aguirre-Ghiso JA, et al: Guidelines for the use and interpretation of assays for monitoring autophagy. Autophagy 8: 445-544, 2012.

19. Levine B: Cell biology: Autophagy and cancer. Nature 446: 745-747, 2007.

20. Pankiv S, Clausen TH, Lamark T, Brech A, Bruun JA, Outzen H, Øvervatn A, Bjørkøy G and Johansen T: p62/SQSTM1 binds directly to Atg8/LC3 to facilitate degradation of ubiquitinated protein aggregates by autophagy. J Biol Chem 282: 24131-24145, 2007.

21. Kepp O, Galluzzi L, Lipinski M, Yuan J and Kroemer G: Cell death assays for drug discovery. Nat Rev Drug Discov 10: 221-237, 2011.

22. Wang J, Whiteman MW, Lian H, Wang G, Singh A, Huang D and Denmark T: A non-canonical MEK/ERK signaling pathway regulates autophagy via regulating Beclin 1. J Biol Chem 284: 21412-21424, 2009

23. Wu YC, Wu WK, Li Y, Yu L, Li ZJ, Wong CC, Li HT, Sung JJ and Cho $\mathrm{CH}$ : Inhibition of macroautophagy by bafilomycin A1 lowers proliferation and induces apoptosis in colon cancer cells. Biochem Biophys Res Commun 382: 451-456, 2009.

24. Fulda S: Targeting extrinsic apoptosis in cancer: Challenges and opportunities. Semin Cell Dev Biol 39: 20-25, 2015.

25. Reed JC: Apoptosis-targeted therapies for cancer. Cancer Cell 3: $17-22,2003$

26. Galluzzi L, Bravo-San Pedro JM, Vitale I, Aaronson SA, Abrams JM, Adam D, Alnemri ES, Altucci L, Andrews D, Annicchiarico-Petruzzelli M, et al: Essential versus accessory aspects of cell death: Recommendations of the NCCD 2015. Cell Death Differ 22: 58-73, 2015.

27. Gewirtz DA: Cytoprotective and nonprotective autophagy in cancer therapy. Autophagy 9: 1263-1265, 2013.

28. Gewirtz DA: The four faces of autophagy: Implications for cancer therapy. Cancer Res 74: 647-651, 2014.

29. Fulda S and Kögel D: Cell death by autophagy: Emerging molecular mechanisms and implications for cancer therapy. Oncogene 34: 5105-5113, 2015.

30. Li Z, Wang J and Yang X: Functions of autophagy in pathological cardiac hypertrophy. Int J Biol Sci 11: 672-678, 2015.

31. Galluzzi L, Pietrocola F, Levine B and Kroemer G: Metabolic control of autophagy. Cell 159: 1263-1276, 2014.

32. Pupyshev AB: Reparative autophagy and autophagy death of cells. Functional and regulatory aspects. Tsitologiia 56: 179-196, 2014 (In Russian).

33. Pinho SS, Carvalho S, Marcos-Pinto R, Magalhães A, Oliveira C, Gu J, Dinis-Ribeiro M, Carneiro F, Seruca R and Reis CA: Gastric cancer: Adding glycosylation to the equation. Trends Mol Med 19: 664-676, 2013. 
34. Beheshti Zavareh R, Lau KS, Hurren R, Datti A, Ashline DJ, Gronda M, Cheung P, Simpson CD, Liu W, Wasylishen AR, et al: Inhibition of the sodium/potassium ATPase impairs $\mathrm{N}$-glycan expression and function. Cancer Res 68: 6688-6697, 2008.

35. Contessa JN, Bhojani MS, Freeze HH, Ross BD, Rehemtulla A and Lawrence TS: Molecular imaging of N-linked glycosylation suggests glycan biosynthesis is a novel target for cancer therapy. Clin Cancer Res 16: 3205-3214, 2010.
36. Vitale I, Manic G, Dandrea V and De Maria R: Role of autophagy in the maintenance and function of cancer stem cells. Int J Dev Biol 59: 95-108, 2015.

37. Hoppener JW, De Wit MJ, Simarro-Doorten AY, Roijers JF, van Herrewaarden HM, Lips CJ, Parente F, Quincey D, Gaudray $\mathrm{P}$, Khodaei $\mathrm{S}$, et al: A putative human zinc-finger gene (ZFPL1) on 11q13, highly conserved in the mouse and expressed in exocrine pancreas. The European Consortium on MEN 1. Genomics 50: 251-259, 1998. 